\title{
Boson Fields with Nonlinear Selfinteraction in Two Dimensions*
}

\author{
JAMES GLIMM \\ Mass. Institute of Technology, Cambridge, Mass., \\ Received December 15, 1967
}

\begin{abstract}
Semiboundedness of the total Hamiltonian is proved for a selfinteracting Boson field in two dimensional space time. The interaction is given by a Wick polynomial : $P(\Phi)$ : . The polynomial $P$ is required to have even degree and its leading coefficient must be positive. A space cutoff is introduced in the interaction Hamiltonian.
\end{abstract}

\section{§1. Introduction}

In [10] NELSON considered the following problem. Let $\Phi$ be a neutral scalarfield of mass $\mu_{0}>0$ in two dimensional space time and let

$$
H=H_{0}+g \int: \Phi^{4}(x): d x,
$$

where $H_{0}$ is the free Hamiltonian for the mass $\mu_{0}$ and $g>0$. If the system is placed in a box with periodic boundary conditions then Nelson proved that $H$ is bounded from below. $H$ thus has a natural selfadjoint semibounded extension (the Friedrichs extension), which can (presumably) be used to solve the Schrödinger equation. In [5], Jaffe considered the related Hamiltonian

$$
H=H_{0}+\int: P(\Phi(x)): h(x) d x,
$$

$P$ a polynomial, again in two dimensional space time. Jaffe showed $H$ to be a symmetric densely defined operator; no box is needed here. In this paper we apply Nelson's method to Jaffe's Hamiltonian (1.2). Our main result is

Theorem A. Let $h$ be a nonnegative function in $L_{1} \cap L_{2}$. Suppose that the polynomial $P$ in (1.2) has even degree and that the leading coefficient is positive. Then $H$ is bounded from below.

By elementary methods we also show that the Hamiltonian

$$
\varepsilon N+\int: \Phi^{2}(x): h^{2}(x) d x
$$

is bounded from below, where $\varepsilon$ is any positive number and $N$ is the number of particles operator. This bound on (1.3) permits an improve-

* This work was supported in part by the National Science Foundation, NSF GP 7477 
ment in the results of [2]. In [1], we defined a renormalized Hamiltonian $H_{\text {ren }}$ for Fermi and Boson fields with Yukawa coupling (and with a space cutoff). The Hamiltonian was shown to exist as a bilinear form. In [2] we showed that

$$
0 \leqq 2^{-1} F_{\tau} \leqq H_{\text {ren }}+\delta \int: \Phi^{2}(x): h^{2}(x) d x+c I .
$$

Here $F_{\tau}$ is an operator defined in [2], and all that concerns us is its property:

$$
N \leqq F_{\tau} .
$$

Also $\delta$ is a number which may be taken as small as desired, but is bounded above, $\delta \leqq \delta_{0}$, and in general $\delta_{0}$ is negative. Combining the bound on (1.3) with (1.4) and (1.5), we have the following theorem.

Theorem B. The bilinear form $H_{\mathrm{ren}}$ of [2] is bounded from below. Furthermore for any $a$ in $\left[0,2^{-1}\right)$ and for any number $\delta$ there is $a c=c(a, \delta)$ such that

$$
0 \leqq a F_{\tau} \leqq H_{\text {ren }}+\delta \int: \Phi^{2}(x): h^{2}(x) d x+c I .
$$

Thus the upper bound on the $\delta$ in (1.4) is removed and any choice of $\delta$ is possible. If our model were realistic ( $h \equiv 1$, four dimensions) we would then choose $\delta$ so that the spectrum of the operator on right side of (1.6) agrees with experiment. See [7] for a further discussion of this point. One would like to know how the spectrum depends on $\delta$. The idea expressed in $[1$, p. 345] on the removal of the space cutoff was first advanced by GUENIN [3]. GUENIN proposed that if $A$ is an observable associated with a bounded region and $h \equiv 1$ on a larger region then

$$
e^{-i t H} A e^{i t H}
$$

is (formally) independent of $h$ for small $t$. See [12, Theorem 3] for a further development of this point of view.

Segal [12] and SymanzIK (unpublished) have studied Hamiltonians such as (1.2). As part of a general study of Wick products, $[12,13]$, SEgAL has announced a new proof of Jaffe's theorem that (1.2) is densely defined. Symanzik observed that Nelson's methods were not limited to fourth powers in the interaction and that the periodic boundary conditions in a finite region (as used by NeLSON) could be replaced by other, for example Dirichlet, conditions.

In Theorem A, $H_{0}$ could be replaced by $N$ with only trivial changes in the proof.

\section{§ 2. A Domain for $\mathbf{H}$}

We use the Fock space representation for our field $\Phi$. The Fock Hilbert space $\mathscr{F}$ is a direct sum

$$
\mathscr{F}=\sum_{n=0}^{\infty} \oplus \mathscr{F}_{n}
$$


and $\mathscr{F}_{n}$ is the space of symmetric square integrable functions of $n$ variables. Let

Then

$$
\mu(k)=\left(\mu_{0}^{2}+k^{2}\right)^{1 / 2}
$$

$$
\begin{aligned}
& \Phi^{-}(x)=\int e^{i k x} a(k) \mu(k)^{-1 / 2} d k \\
& \Phi^{+}(x)=\int e^{i k x} a^{*}(-k) \mu(k)^{-1 / 2} d k
\end{aligned}
$$

and $\Phi=\Phi^{-}+\Phi^{+}$, where $a$ and $a^{*}$ are the annihilation and creation operators,

By definition,

$$
\left[a(k), a^{*}\left(k^{\prime}\right)\right]=\delta\left(k-k^{\prime}\right)
$$

$$
: \Phi^{p}(x):=\sum_{j}\left(\begin{array}{c}
p \\
j
\end{array}\right) \Phi^{+}(x)^{j} \Phi^{-}(x)^{p-j},
$$

or in other words, the Wick product differs from the ordinary product in that all the annihilators are placed to the right and the creators are placed to the left. : $\Phi^{p}(x)$ : is not an operator, but it is a densely defined bilinear form.

We take Fourier transforms to compute

$$
\begin{aligned}
\int: \Phi^{p}(x): h(x) & d x \\
& =\sum_{j}\left(\begin{array}{l}
p \\
j
\end{array}\right) \int a^{*}\left(-k_{1}\right) \ldots a^{*}\left(-k_{j}\right) a\left(k_{j+1}\right) \ldots a\left(k_{p}\right) \\
& \times \hat{h}\left(k_{1}+\cdots+k_{p}\right) \Pi \mu\left(k_{i}\right)^{-1 / 2} d k_{i}
\end{aligned}
$$

where $\hat{h}$ is the Fourier transform of $h$. We assume $h$ is in $L_{2}$, and so $\hat{h}$ is in $L_{2}$ also. Since $\mu(k) \sim|k|$ for large $k$, one can show that

$$
\hat{h}\left(k_{1}+\cdots+k_{p}\right) \prod_{i} \mu\left(k_{i}\right)^{-1 / 2} \in L_{2} \text {. }
$$

It is well known that (2.6) implies that each integral on the right side of (2.5) is an operator defined on the domain $\mathscr{D}\left(N^{p / 2}\right)$ of $N^{p / 2}$. This domain is the set of $\phi=\phi_{0}, \phi_{1}, \ldots, \phi_{j} \in \mathscr{F}_{j}$

with

$$
\sum\left\|n^{p / 2} \phi_{n}\right\|^{2}<\infty .
$$

Thus (2.5) is an operator defined on $\mathscr{D}\left(N^{p / 2}\right)$. Similarly

$$
H_{0}+\int: P(\Phi(x)): h(x) d x
$$

is an operator defined on the dense domain, $\mathscr{D}\left(H_{0}\right) \cap \mathscr{D}\left(N^{d / 2}\right)$, where $d$ is the degree of the polynomial $P$.

\section{§ 3. $\varepsilon N+\int: \Phi^{2}(x): h^{2}(x) d x$ is positive}

In this section we suppose $h^{2} \in L_{2}$ so that the operator (1.3) is defined on $\mathscr{D}(N)$. We set

$$
\Phi(k)=a(k)+a^{*}(-k)
$$


Then

$$
\begin{aligned}
& \int: \Phi^{2}(x): h^{2}(x) d x \\
= & \int \hat{h}^{2}(k+l) \Phi(k) \Phi(l) \mu(k)^{-1 / 2} \mu(l)^{-1 / 2} d k d l \\
= & \int \hat{h}(\eta+k) \hat{h}(-\eta+l) \Phi(k) \Phi^{*}(-l) \mu(k)^{-1 / 2} \mu(l)^{-1 / 2} d k d l d \eta .
\end{aligned}
$$

We assume $h$ is real, so that $\hat{h}^{-}(k)=\hat{h}(-k)$. Then (3.1) is equal to

where

$$
\int: A(\eta) A^{*}(\eta): d \eta
$$

$$
A(\eta)=\int \hat{h}(\eta+k) \Phi(k) \mu(k)^{-1 / 2} d k
$$

Let

and set (3.1) equal to

$$
B_{\varrho}=\int_{|\eta| \leqq \varrho}: A(\eta) A^{*}(\eta): d \eta
$$

$$
B_{\varrho}+B_{\varrho}^{\prime} .
$$

We assert that $B_{\varrho}$ is semibounded and that $B_{\varrho}^{\prime}$ is small relative to $N$, for large $\varrho$. The second statement means that

$$
B_{\varrho}^{\prime} \leqq \varepsilon(N+I)
$$

if $\varrho \geqq \varrho_{0}=\varrho_{0}(\varepsilon)$. To prove (3.3) we note that $B_{\varrho}^{\prime}$ can be written as a sum of four integrals of the form

$$
B=\int v\left(k_{1}, k_{2}\right) a^{\#}\left(k_{1}\right) a^{\#}\left(k_{2}\right) d k_{1} d k_{2},
$$

with $a^{\#}=a^{*}$ or $a^{\#}=a$ and with $v \in L_{2}$. Moreover $v$ depends on $\varrho$ and $\|v\|_{2} \rightarrow 0$ as $\varrho \rightarrow \infty$. It is known that

$$
(N+I)^{-1 / 2} B(N+I)^{-1 / 2} \leqq \text { const. }\|v\|_{2} I,
$$

and so (3.3) follows.

To prove that $B_{e}$ is semibounded we use the commutation relations (2.3) to remove the Wick ordering from (3.2). We find

and so

$$
: A(\eta) A^{*}(\eta):=A(\eta) A^{*}(\eta)-\int|\hat{h}(\eta+k)|^{2} \mu(k)^{-1} d k I
$$

$$
B_{e}=\int_{|\eta| \leqq \varrho} A(\eta) A^{*}(\eta) d \eta+\iint_{|\eta| \leqq \varrho}|\hat{h}(\eta+k)|^{2} \mu(k)^{-1} d k d \eta I .
$$

The first term on the right is obviously positive and the second is a finite multiple of the identity. Thus $B_{\varrho}$ and also

$$
\varepsilon N+\int: \Phi^{2}(x) h^{2}(x) d x
$$

are semibounded under the assumption that $h^{2}$ is nonnegative and in $L_{2}$.

\section{\$ 4. Reduction to a Problem with Discrete Momentum}

We follow a procedure of NELSON [9] for approximating (2.5) by a finite sum. 
Choose numbers $\gamma$ and $\varkappa$. (Later we let $\gamma \rightarrow 0, \varkappa \rightarrow \infty$ ). We define

and

$$
\begin{aligned}
\Gamma & =\{n \gamma: n=0, \pm 1, \ldots\} \\
\Gamma_{\varkappa} & =\{k: k \in \Gamma,|k| \leqq x\}
\end{aligned}
$$

$$
a_{\gamma}^{\#}(k)=\gamma^{-1 / 2} \int_{0}^{\gamma} a^{\#}(k+l) d l
$$

where $a^{\#}$ equals $a$ or $a^{*}$ and $l \in \Gamma$. Then

Let

$$
\left[a_{\nu}(k), a_{\gamma}^{*}(l)\right]=\delta_{k l}= \begin{cases}1 & \text { if } k=l \\ 0 & \text { otherwise }\end{cases}
$$

$$
H_{0 \gamma \varkappa}=\sum_{k \in \Gamma_{\varkappa}} \mu(k) a_{\gamma}^{*}(k) a_{\gamma}(k) .
$$

One can check that each $\phi$ in $\mathscr{D}\left(H_{0}\right)$ is in $\mathscr{D}\left(H_{0 \gamma x}\right)$ also and that

$$
\lim _{\substack{\gamma \rightarrow 0 \\ x \rightarrow \infty}} H_{0 \gamma x} \phi=H_{0} \phi \text {. }
$$

Next we approximate (2.5) by

where

$$
\begin{aligned}
: \Phi_{\gamma \varkappa}^{p}(h):= & \gamma^{p / 2} \sum_{j=0}^{p}\left(\begin{array}{l}
p \\
j
\end{array}\right) \sum_{k_{i} \in \Gamma_{\varkappa}} a_{\gamma}^{*}\left(-k_{1}\right) \ldots a_{\gamma}^{*}\left(-k_{j}\right) \\
& \times a_{\gamma}\left(k_{j+1}\right) \ldots a_{\gamma}\left(k_{p}\right) \hat{h}_{\nu}\left(\sum_{i}\left[k_{i}\right]\right) \prod_{i} \mu\left(\left[k_{i}\right]\right)^{-1 / 2}
\end{aligned}
$$

and

$$
\hat{h}_{\gamma}(k)=\int_{-\pi / \gamma}^{\pi / \gamma} e^{i k x} h(x) d x
$$

$$
[k]=\sup \{l: l \in \Gamma, l \leqq k\}
$$

is the integral part of $k$ relative to the lattice $\Gamma$. Since $\mathrm{h} \in L_{1}, \hat{h}$ is continuous and

$$
\hat{h}_{\nu}\left(\sum\left[k_{i}\right]\right) \prod_{i} \mu\left(\left[k_{i}\right]\right)^{-1 / 2} \rightarrow \hat{h}\left(\sum k_{i}\right) \prod_{i} \mu\left(k_{i}\right)^{-1 / 2}
$$

uniformly. Let $\mathscr{D}$ be the set of states $\phi=\left\{\phi_{0}, \phi_{1}, \ldots\right\}$ with $\phi_{n}\left(k_{1}, \ldots, k_{n}\right)=0$ for $n$ or $\Sigma\left|k_{i}\right|$ large. If $\phi$ and $\psi$ are in $\mathscr{D}$ then

$$
\lim _{\substack{\gamma \rightarrow 0 \\ x \rightarrow \infty}}\left(\psi,: \Phi_{\gamma \varkappa}^{p}(h): \phi\right)=\left(\psi, \int: \Phi^{p}(x): h(x) d x \phi\right)
$$

Thus the bilinear form of

$$
H_{\gamma \varkappa}=H_{0 \gamma \varkappa}+\sum b_{p}: \Phi_{\gamma \varkappa}^{p}(h):
$$

converges to $H$ on $\mathscr{D} \times \mathscr{D}$ where $b_{0}, b_{1}, \ldots$ are the coefficients of $x^{0}, x^{1}, \ldots$ in the polynomial $P(x)$. Hence if the $H_{\gamma x}$ are semibounded with a lower bound independent of $\gamma$ and $\varkappa$ then $H$ is semibounded also. 
Let $\mathscr{F}_{\gamma}$ be the subspace of $\mathscr{F}$ consisting of functions which are piecewise constant between lattice points. In other words,

$$
\begin{gathered}
\phi=\phi_{0}, \phi_{1}, \ldots \in \mathscr{F}_{\gamma} \text { if } \\
\phi_{n}\left(k_{1}, \ldots, k_{n}\right)=\phi\left(\left[k_{1}\right], \ldots,\left[k_{n}\right]\right) .
\end{gathered}
$$

Let $\mathscr{F}_{\gamma \chi}$ be the subspace of $\mathscr{F}_{\gamma}$ defined by the restriction

$$
\phi_{n}\left(k_{1}, \ldots, k_{n}\right)=0 \text { if }\left[k_{i}\right] \notin \Gamma_{x}
$$

for some $i, 1 \leqq i \leqq n$.

The operators $a_{\gamma}^{*}(k)$ and $a_{\gamma}(k), k \in \Gamma_{x}$, leave $\mathscr{F}_{\gamma x}$ invariant and act irreducibly on $\mathscr{F}_{\gamma x}$. We set $\gamma=2^{-v}, x=2^{\nu}$, and observe that $\mathscr{F}_{2}{ }^{-\nu}, 2^{\nu}$ increases monotonically with $v$ and that

is dense in $\mathscr{F}$ and

$$
\mathscr{D}^{\prime}=\mathscr{D} \cap \bigcup_{\nu} \mathscr{F}_{2}{ }^{-\nu}, 2^{\nu}
$$

$$
H \subset\left(H \mid \mathscr{D}^{\prime}\right)^{-} \text {. }
$$

Here $H \mid \mathscr{D}^{\prime}$ denotes the restriction of $H$ to $\mathscr{D}^{\prime}$. Thus it is sufficient to prove the semiboundedness of

$$
H_{\gamma x} \mid\left(\mathscr{D}\left(H_{0}\right) \cap \mathscr{D}\left(N^{d / 2}\right) \cap \mathscr{F}_{\gamma x}\right)
$$

with a lower bound independent of $\gamma$ and $\varkappa$.

\section{$\S$ 5. Diagonalizing the Potential}

So far we have used a representation of the Hilbert space in which $H_{0}$ is diagonalized, or is represented as a multiplication operator. In this section we give a new representation of $\mathscr{F}_{\gamma x}$ in which the interaction term : $\Phi^{p}(h)$ : is a multiplication operator while the free Hamiltonian becomes more complicated. Let

$$
\begin{aligned}
q_{|k|} & =(1 / 4 \mu(k))^{1 / 2}\left[a_{\gamma}(k)+a_{\gamma}^{*}(k)+a_{\gamma}(-k)+a_{\gamma}^{*}(-k)\right] \\
q_{-|k|} & =i(1 / 4 \mu(k))^{1 / 2}\left[-a_{\gamma}(|k|)+a_{\gamma}^{*}(|k|)+a_{\gamma}(-|k|)-a_{\gamma}^{*}(-|k|)\right] \\
p_{|k|} & =i(\mu(k) / 4)^{1 / 2}\left[a_{\gamma}(k)-a_{\gamma}^{*}(k)+a_{\gamma}(-k)-a_{\gamma}^{*}(-k)\right] \\
p_{-|k|} & =(\mu(k) / 4)^{1 / 2}\left[a_{\gamma}(|k|)+a_{\gamma}^{*}(|k|)-a_{\gamma}(-|k|)-a_{\gamma}^{*}(-|k|)\right]
\end{aligned}
$$

for $0 \neq k \in \Gamma$ and let

One can compute that

$$
\begin{aligned}
& q_{0}=\left(1 / 2 \mu_{0}\right)^{1 / 2}\left[a_{\gamma}(0)+a_{\gamma}^{*}(0)\right] \\
& p_{0}=i\left(\mu_{0} / 2\right)^{1 / 2}\left[a_{\gamma}(0)-a_{\gamma}^{*}(0)\right] .
\end{aligned}
$$

$$
H_{0 \gamma x}=\sum_{k \in \Gamma_{x}} 2^{-1}\left[p(k)^{2}+\mu(k)^{2} q(k)^{2}-\mu(k)\right] .
$$

As in $[9, \S 3-4]$ we replace $p_{k}$ and $q_{k}$ by unitarily equivalent operators. Let

$$
\mathscr{H}_{\gamma x}=\underset{k \in \Gamma_{x}}{\otimes} \mathscr{H}_{k} .
$$


where $\mathscr{H}_{k}$ is $L_{2}$ of the real line with respect to the Gaussian measure

$$
\phi_{k}^{2}(q) d q=(\mu(k) / \pi)^{1 / 2} \exp \left(-\mu(k) q^{2}\right) d q .
$$

There is a unitary equivalence between $\mathscr{H}_{\gamma x}$ and $\mathscr{F}_{\gamma x}$ which sends $q_{k}$ into multiplication by $q$ in the factor $\mathscr{H}_{k}$ and $p_{k}$ into the operator

$$
\phi_{k}^{-1} i(d / d q) \phi_{k}
$$

again acting in the factor $\mathscr{H}_{k}$. The proof of this statement is essentially von Neumann's uniqueness theorem for irreducible representations of the commutation relations. We identify $\mathscr{H}_{\gamma x}$ and $\mathscr{F}_{\gamma x}$ and we identify $q_{k}$, etc. with its image, multiplication by $q$, etc. Let

$$
\begin{aligned}
H_{\mu(k)} & =2^{-1} \phi_{k}^{-1}\left[-(d / d q)^{2}+\mu(k) q^{2}-\mu(k)\right] \phi_{k} \\
& =-2^{-1}(d / d q)^{2}+\mu(k) q(d / d q),
\end{aligned}
$$

acting on $\mathscr{H}_{k}$. Now $-\mathscr{H}_{\mu(k)}$ is the infinitesimal generator of a known Markoff process and furthermore the operator $e^{-t H_{\mu(k)}}$ is an integral operator and the kernel can be computed explicitly, [10]. In particular

$$
\left(e^{-t B_{\mu(k)}} \psi\right)(q)=\int p^{t}\left(q, q^{\prime}\right) \psi\left(q^{\prime}\right) \phi_{k}^{2}\left(q^{\prime}\right) d q^{\prime}
$$

for $\psi \in \mathscr{H}_{k}$, where

$$
p^{t}\left(q, q^{\prime}\right)=\left(1-e^{-2 \mu t}\right)^{-1 / 2} \exp \left[-\frac{\mu\left(q^{\prime}-e^{-\mu t} q\right)^{2}}{1-e^{-2 \mu t}}+\mu q^{\prime 2}\right]
$$

Let $q$ now denote a variable in a Euclidean space $R_{\varkappa}$ and let $q$ have coordinates $q_{k}, k \in \Gamma_{*}$. Then

$$
\phi_{\varkappa}^{2}(q) d q=\prod_{k \in \Gamma_{\varkappa}} \phi_{\varkappa}^{2}\left(q_{k}\right) d q_{k}
$$

is the product of the measures (5.2) and

$$
H_{\gamma \varkappa}=L_{2}\left(\phi_{\varkappa}^{2}(q) d q\right) \text {. }
$$

In addition to the function space $L_{2}$, we will have to consider

$$
L_{r}=L_{r}\left(\phi_{\varkappa}^{2}(q) d q\right) \text {. }
$$

Since $\int \phi_{x}^{2}(q) d q=1$, we have $L_{r_{2}} \subset L_{r_{1}}$ if $r_{1} \leqq r_{2}$.

Lemma 5.1. $\exp \left(-t H_{0 \gamma x}\right)$ is a contraction operator on $L_{r}, 1 \leqq r \leqq \infty$. When $T \leqq t, 1<p$ and $r<\infty$ it is a contraction from $L_{p}$ to $L_{r}$, for some $T$ not depending on $\gamma$ or $x$. If $p$ is bounded away from one and $r$ is bounded then $T$ does not depend on $p$ or $r$.

Proof. If $\psi \in L_{\infty}$, then by a change of variables,

$$
\begin{aligned}
& \left|\left(e^{-t H_{\mu}} \psi\right)(q)\right| \\
& \quad \leqq(\mu / \pi)^{1 / 2}\left(1-e^{-2 \mu t}\right)^{-1 / 2} \int \exp \left[\frac{-\mu\left(q_{k}^{\prime}-e^{-2 \mu t} q_{k}\right)^{2}}{1-e^{-2 \mu t}}\right] \psi\left(q^{\prime}\right) d q_{k}^{\prime} \\
& \quad \leqq \pi^{-1 / 2}\|\psi\|_{\infty} \int \exp \left(-q_{k}^{\prime 2}\right) d q_{k}^{\prime}=\|\psi\|_{\infty},
\end{aligned}
$$

and so $\exp \left(-t H_{0 \gamma \varkappa}\right)$ is a contraction on $L_{\infty}$. Since $H_{0 \gamma \varkappa} \geqq 0, \exp \left(-t H_{0 \gamma \alpha}\right)$ 
is a contraction on $L_{2}$ and thus on $L_{r}, 2 \leqq r \leqq \infty$, by the Riesz Thorin convexity theorem [8]. Let $\left\|\exp \left(-t H_{0 \gamma x}\right)\right\|_{p r}=C_{p r}$ be the norm of $\exp \left(-t H_{0 y x}\right)$ as an operator from $L_{p}$ to $L_{r}$. The cited theorem states that $\log C_{p r}$ is a convex function of $p^{-1}$ and $r^{-1}$ in the square

$$
0 \leqq p^{-1}, r^{-1} \leqq 1 \text {. }
$$

Now $\exp \left(-t H_{0 \gamma x}\right)$ is symmetric in the $L_{2}$ inner product, since $p^{t}\left(q_{k}, q_{k}^{\prime}\right)=\exp \left[-b\left(e^{-\mu t} q_{k}^{\prime 2}-2 q_{k} q_{k}^{\prime}+e^{-\mu t} q_{k}^{2}\right)\right]$ with

$$
b=\mu e^{-\mu t}\left(1-e^{-2 \mu t}\right)^{-1} \text {. }
$$

Thus by duality, $\exp \left(-t H_{0 \gamma x}\right)$ is a contraction from $L_{r}$ to $L_{r}, 1<r \leqq 2$. It is a contraction on $L_{1}$ by taking limits as $r \rightarrow 1$.

Let

Then

$$
a^{2}\left(q_{k}\right)=\int p^{t}\left(q_{k}, q_{k}^{\prime}\right)^{2} \phi_{k}^{2}\left(q_{k}\right) d q_{k} .
$$

$$
\left|\left(e^{-t H_{\mu}} \psi\right)\left(q_{k}\right)\right| \leqq a\left(q_{k}\right)\|\psi\|_{2}
$$

by the Schwartz inequality, so $\left\|a\left(q_{k}\right)\right\|_{r}$ bounds the norm of $\exp \left(-t H_{\mu}\right)$ as an operator from $L_{2}$ to $L_{r}$. For $r-1<e^{2 \mu t},\left\|a\left(q_{k}\right)\right\| r$ is finite and has a bound independent of $\mu \geqq \mu_{0}$ and $t \geqq T$, for some $T$, as computed in [10]. Let $\mathscr{H}_{k}^{\prime}$ be the orthogonal compliment of 1 in $\mathscr{H}_{k}$. Since the norm of $\exp \left(-t H_{\mu}\right)$, as an operator from $\mathscr{H}_{k}^{\prime}$ to $\mathscr{H}_{k}^{\prime}$, tends to zero as $t \rightarrow \infty$, the norm of

$$
\exp \left(-t H_{\mu}\right): \mathscr{H}_{k}^{\prime} \rightarrow L_{4}
$$

also tends to zero as $t \rightarrow \infty$, uniformly in $k$. Let $\psi^{\prime} \in \mathscr{H}_{k}^{\prime}$ and let $\psi=1+\psi^{\prime}$. Then

$$
\begin{aligned}
\left(\exp \left(-t H_{\mu}\right) \psi\right)^{2} & =1+2 \exp \left(-t H_{\mu}\right) \psi^{\prime}+\left(\exp \left(-t H_{\mu}\right) \psi^{\prime}\right)^{2} \\
& =[1+c]+\left[2 \exp \left(-t H_{\mu}\right) \psi^{\prime}+\left(\exp \left(-t H_{\mu}\right) \psi^{\prime}\right)^{2}-c\right] .
\end{aligned}
$$

We choose

$$
c=\left(1,\left(\exp \left(-t H_{\mu}\right) \psi^{\prime}\right)^{2}\right)=\left\|\exp \left(-t H_{\mu}\right) \psi^{\prime}\right\|_{2}^{2}
$$

and then the terms in the brackets above are orthogonal. Thus

For large $t$,

$$
\begin{aligned}
\left\|\exp \left(-t H_{\mu}\right) \psi\right\|_{4}^{4} & =\left\|\left(\exp \left(-t H_{\mu}\right) \psi\right)^{2}\right\|_{2}^{2} \\
& \leqq(1+c)^{2}+\left(2 c^{1 / 2}+\left\|\left(\exp \left(-t H_{\mu}\right) \psi^{\prime}\right)^{2}\right\|_{2}\right)^{2} \\
& \leqq(1+c)^{2}+8 c+2\left\|\left(\exp \left(-t H_{\mu}\right) \psi^{\prime}\right)^{2}\right\|_{2}^{4} \\
& =(1+c)^{2}+8 c+2\left\|\exp \left(-t H_{\mu}\right) \psi^{\prime}\right\|_{4}^{4} .
\end{aligned}
$$

$$
\left\|\exp \left(-t H_{\mu}\right) \psi\right\|_{4}^{4} \leqq 1+2\left\|\psi^{\prime}\right\|_{2}^{2}+\left\|\psi^{\prime}\right\|_{2}^{4}=\|\psi\|_{2}^{4} .
$$

and so $\exp \left(-t H_{\mu}\right)$ and $\exp \left(-t H_{0 \gamma \alpha}\right)$ are contractions from $L_{2}$ to $L_{4}$. The lower bound $T$ on $t$ does not depend on $\varkappa$ or $\gamma$.

We apply the Riesz Thorin convexity theorem to the maps

$$
\begin{aligned}
& \exp \left(-t H_{0 \gamma \varkappa}\right): L_{2} \rightarrow L_{4} \\
& \exp \left(-t H_{0 \gamma \varkappa}\right): L_{\infty} \rightarrow L_{\infty}
\end{aligned}
$$


and conclude that for large $t$

$$
\exp \left(-t H_{0 \gamma x}\right): L_{r} \rightarrow L_{2 r}
$$

is a contraction, $r \geqq 2$, We take products of several of these maps and conclude that $\exp \left(-t H_{0 \gamma x}\right)$ is a contraction from $L_{2}$ to $L_{r}$ for any $r<\infty$, if $t$ is large. By duality it is a contraction from $L_{p}$ to $L_{2}$ and hence from $L_{p}$ to $L_{r}$ for any $p, r \in(1, \infty)$, and the lower bound on $t$ is independent of $\gamma, x, p$ and $r$ if $p$ is bounded away from 1 and $r$ is bounded.

Now we show that the interaction term $: \phi_{\gamma x}^{p}(h):$ is a polynomial in the $q^{\prime} s$.

Let

$$
\phi_{\gamma \varkappa}(x)=\gamma^{1 / 2} \sum_{k \in \Gamma_{x}}\left(a_{\gamma}(k)+a_{\gamma}^{*}(-k)\right) \mu(k)^{-1 / 2} e^{i k x}
$$

Since

$$
\left(a_{\gamma}(k)+a_{\gamma}^{*}(-k)\right) \mu(k)^{-1 / 2}=\left\{\begin{array}{lll}
q_{|k|}+i q_{-|k|} & \text { if } & k>0 \\
2^{1 / 2} q_{0} & \text { if } k=0 \\
q_{|k|}-i q_{-|k|} & \text { if } k<0
\end{array}\right.
$$

$\phi_{\gamma \varkappa}(x)$ and also $\phi_{x \gamma}^{p}(x)$ are polynomials in the $q^{\prime} s$. We use the formula

$$
\phi_{\gamma \varkappa}^{p}(x)=\sum_{j=0}^{[p / 2]} \frac{p !}{(p-2 j) ! j !} 2^{-j} c_{\varkappa}^{j}: \phi_{\gamma \varkappa}^{p-2 j}(x):
$$

to conclude by induction on $p$ that $: \phi_{\gamma x}^{p}(x)$ : is also a polynomial in the $q^{\prime} s$. In (5.8) the coefficient

$$
\frac{p !}{(p-2 j) ! j !} 2^{-j}
$$

is just the number of ways of selecting $j$ unordered pairs from $p$ objects and $c_{\varkappa}$ is defined by the formula

we have the bound

$$
c_{\varkappa}=\gamma \sum_{k \in \Gamma_{\varkappa}} \mu(k)^{-1}
$$

$$
c_{\varkappa} \leqq K_{1} \ln \varkappa
$$

where $K_{1}$ is independent of $\gamma$ and $\varkappa$. Thus

$$
: \phi_{\gamma \varkappa}^{p}(h):=\int_{-\pi / \gamma}^{\pi / \gamma}: \phi_{\gamma \varkappa}^{p}(x): h(x) d x
$$

is a polynomial in the $q^{\prime} s$, as desired.

Let

$$
P(x)=b_{0}+b_{1} x+\cdots+b_{d} x^{d}
$$

be the polynomial in (1.2) and let

$$
V_{\gamma \varkappa}=\sum b_{p}: \phi_{\gamma \varkappa}^{p}(h):
$$

denote our approximate interaction term, as in (4.6). 
Lemma 5.2. For some constant $K_{2}$, independent of $\gamma$ and $\varkappa$, we have

$$
-(\ln x)^{d / 2} K_{2} \leqq V_{\gamma \varkappa} .
$$

Proof. We use (5.8) to remove the Wick ordering in (5.10) and obtain

$$
V_{\gamma x}=\sum a_{p}\left(c_{x}\right) \int_{\pi / \gamma}^{\pi / \gamma} \phi_{\gamma x}^{p}(x) h(x) d x
$$

where $a_{p}$ is a polynomial in $c_{\varkappa}$ of degree at most [(d-p)/2]. The coefficients of $a_{p}$ depend only on the coefficients of $P$, and so we have an estimate

$$
\left|a_{p}\left(c_{\varkappa}\right)\right| \leqq K^{\prime} c_{\varkappa}^{[(d-p) / 2]}
$$

Since $a_{d}=b_{d}>0$ and since $d$ is even by hypothesis, it follows that $0<\sum a_{p}\left(c_{x}\right) x^{p}$ for $K^{\prime \prime}\left(c_{x}+1\right)<|x|^{2}$ and

$$
-c_{x}^{d / 2} K^{\prime \prime \prime} \leqq \sum a_{p}\left(c_{x}\right) x^{p}
$$

for all $x$. We bound $c_{x}$ by (5.9) and the proof is complete.

Lemma 5.3. $V_{\gamma \varkappa} \in L_{r}$ for all $r<\infty$ and if $\lambda \leqq \varkappa$

$$
\left\|V_{\gamma \alpha}-V_{\gamma \lambda}\right\|_{2 j}^{2 j} \leqq(d j) ! K_{3}^{j} \lambda-^{-j},
$$

where $K_{3}$ is a constant which is independent of $\gamma, \lambda$ and $x$.

Proof. We use the particle representation, $\mathscr{F}_{\gamma \kappa}$, in place of the representation $\mathscr{H}_{\gamma x}=L_{2}\left(\phi_{k}^{2} d q\right)$. Now $1 \in \mathscr{H}_{\gamma x}$ corresponds to the vacuum state

$$
\Omega=1,0, \ldots \in \mathscr{F}_{\gamma x},
$$

so

$$
\begin{aligned}
\left\|V_{\gamma \varkappa}-V_{\gamma \lambda}\right\|_{2 j}^{2 j} & =\int\left(V_{\gamma \varkappa}-V_{\gamma \lambda}\right)^{2 j} \phi_{k}^{2} d q \\
& =\left(\left(V_{\gamma \varkappa}-V_{\gamma \lambda}\right)^{j} \Omega,\left(V_{\gamma \varkappa}-V_{\gamma \lambda}\right)^{j} \Omega\right) \\
& \left.=\| V_{\gamma \varkappa}-V_{\gamma \lambda}\right)^{j} \Omega \|^{2} .
\end{aligned}
$$

We set $\lambda=0$ above and get

$$
\left\|V_{\gamma x}\right\|_{2 j}^{2 j}=\left\|V_{\gamma x}^{2 j} \Omega\right\|^{2}<\infty
$$

and so $V_{\gamma x} \in L_{r}$ for all $r<\infty$. We return to (5.13) and note that $V_{\gamma x}-V_{\gamma \lambda}$ is a sum of $d 2^{d}$ terms of the form

$$
A=b_{p} \gamma^{p / 2} \sum_{k_{i}}\left[\hat{h}_{\gamma}\left(\sum_{i=1}^{p} k_{i}\right): \prod_{i=1}^{p}\left(a_{\gamma}^{\#}\left( \pm k_{i}\right) \mu\left(k_{i}\right)^{-1 / 2}\right):\right]
$$

where in the summation over $k_{i}$ we have

$$
k_{i} \in \Gamma_{\varkappa} \text { for } 1 \leqq i \leqq p, p \leqq d, \quad \text { and } \quad k_{i} \notin \Gamma_{\lambda}
$$

for at least one $i$. Summing again over the same range of $k_{i}$, we have

$$
\gamma^{p} \sum_{k_{i}}\left[\left|\hat{h}_{\lambda}\left(\sum_{i=1}^{p} k_{i}\right)\right|^{2} \prod_{i=1}^{p} \mu\left(k_{i}\right)^{-1}\right] \leqq K_{4} \lambda^{-1}
$$

and $K_{4}$ is independent of $\lambda, \varkappa$ and $\gamma$. 
Let $\psi$ be a state with at most $l$ particles. It follows from (5.15) and the form of $A$ that

$$
\left.\|A \psi\|^{2} \leqq(l+p) ! / l !\right) K_{4} \lambda^{-1}\|\psi\|^{2}
$$

and furthermore $A \psi$ is a state with at most $l+p$ particles. Thus if we have operators $A_{1}, \ldots, A_{j}$ of the form (5.14),

Hence

$$
\left\|A_{1} \ldots A_{j} \Omega\right\|^{2} \leqq(d j) ! K_{4}^{j} \lambda^{-1} .
$$

$$
\left\|\left(V_{\gamma \varkappa}-V_{\gamma \lambda}\right)^{j} \Omega\right\|^{2} \leqq(d j) !\left(d 2^{d} K_{4}\right)^{j} \lambda^{-j}=(d j) ! K_{3}^{j} \lambda^{-j},
$$

and the proof is complete.

\section{§ 6. Path space}

Let $C$ be the space of continuous paths $q=q(s)$,

$$
q(s) \in R_{\varkappa}, 0 \leqq s<\infty .
$$

There is a measure on $C$ intrinsically associated with the semigroup $\exp \left(-t H_{0 \gamma x}\right)$. To define this measure we set

$$
p_{k}^{t}\left(q_{k}, q_{k}^{\prime}\right) \phi_{k}^{2}\left(q_{k}^{\prime}\right) d q_{k}^{\prime}=\operatorname{Pr}\left\{q_{k}(t)=q_{k}^{\prime} \mid q_{k}(0)=q_{k}\right\},
$$

the probability that $q_{k}(t)=q_{k}^{\prime}$ if it is known that $q_{k}(0)=q_{k} \cdot p_{k}^{t}$ is defined by (5.5); we have added a subscript $k$ to indicate the dependence on $\mu=\mu(k)$. Let

$$
p_{\varkappa}^{t}\left(q, q^{\prime}\right)=\prod_{k \in \Gamma_{\varkappa}} p_{k}^{t}\left(q_{k}, q_{k}^{\prime}\right) .
$$

The $\sigma$-field of measurable subsets of $C$ is generated by the sets

$$
q\left(s_{i}\right) \in B_{i}, 1 \leqq i \leqq j,
$$

where $B_{i}$ is a Borel subset of $R_{x}$. The measure of (6.3) is

$\int_{B_{j} \times \cdots \times B_{j}} \prod_{i=2}^{j} p_{x}^{s_{i}-s_{i-1}}\left(q\left(s_{i-1}\right), q\left(s_{i}\right)\right) \phi_{x}^{2}\left(q\left(s_{i}\right)\right) d q\left(s_{i}\right) \phi_{\varkappa}^{2}(q(0)) d q(0)$

if $s_{1}=0<s_{2}<\ldots<s_{j}$. The definition (6.4) is forced by the definition (6.1) together with the Markov character of the process, the stipulation that each coordinate $q_{k}$ of $q$ defines an independent process and the specification of $\phi_{k}^{2}(q) d q$ as the probability distribution of the initial point $q(0)$ of the path $q$.

If $V_{1}, \ldots, V_{j} \in L_{j}\left(R_{x}, \phi_{x}^{2} d Q\right)$ then we compute

$$
\begin{aligned}
& \int \prod_{i} V_{i}\left(q\left(s_{i}\right)\right) d Q=\int V_{1}(q(0)) \phi_{\varkappa}^{2}(q(0)) d q(0) \\
& \quad \cdot\left[\exp \left(\left(s_{0}-s_{1}\right) H_{0 \gamma \varkappa}\right) V_{2} \exp \left(\left(s_{1}-s_{2}\right) H_{0 \gamma \varkappa}\right)\right. \\
& \left.\quad \cdot\left(\ldots\left(V_{j-1} \exp \left(\left(s_{j-1}-s_{j}\right) H_{0 \gamma \varkappa}\right) V_{j}\right) \ldots\right)\right](q(0))
\end{aligned}
$$

and

$$
\left|\int \prod_{i} V_{i}\left(q\left(s_{i}\right)\right) d Q\right| \leqq \prod_{i}\left\|V_{i}\right\|_{j}
$$


using (6.4) and the fact that $\exp \left(-t H_{0 \gamma \alpha}\right)$ is a contraction on $L_{r}$. Furthermore (6.5) and (6.6) remain valid when some of the times $s_{i}$ coincide.

Lemma 6.1. Let $V$ be a polynomial function on $R_{x}$. Then $\int_{0}^{t} V(q(s)) d s \in L_{p}(C, d Q)$ for all $p<\infty$ and

for $j$ an even positive integer.

$$
\left\|\int_{0}^{t} V(q(s)) d s\right\|_{j} \leqq t\|V\|_{j}
$$

Proof. For a given path $q$,

$$
I=\int_{0}^{t} V(q(s)) d s=\lim _{n} \sum_{l}(t / n) V(q(l t / n))
$$

and since each Riemann approximating sum to $I$ is a measurable function of $q, I=I(q)$ is measurable also. Let $I_{n}(q)$ be the $n^{\text {th }}$ Riemann approximating sum to $I$. Then

by (6.6) and

$$
\left|\int I_{n}(q)^{j} d Q\right| \leqq t^{j}\|V\|_{j}^{j}
$$

where $d$ is the degree of $V$ and

$$
|I| \leqq \text { const. } t\left(M_{t}(q)\right)^{d}
$$

$$
M_{t}(q)=\operatorname{Max}_{0 \leqq s \leqq t}|q(s)| \text {. }
$$

Thus the lemma will follow from the Lebesgue bounded convergence theorem once we show that $M \in L_{p}(C, d Q)$ for all $p<\infty$.

However the $d Q$ probability of the set

$$
\left\{\left|q_{k}(t)\right| \leqq a|| q_{k}(0) \mid\right\}
$$

dominates the Wiener probability of the same set if $\left|q_{k}(0)\right| \leqq a$. It follows that the $L_{p}$ norm of $M_{t}$ is dominated by the Wiener $L_{p}$ norm of $M_{t}$. This latter norm is finite by known properties of Wiener measure, $[4$, p. 25,26$]$.

Lemma 6.2. Let $r \in[1,2)$. There is a $T$ independent of $\gamma$ and $x$ such that if $t \geqq T$ and if $\phi$ and $\psi \in L_{2}\left(\phi_{x}^{2} d q\right)$ then $\phi(q(0)) \psi(q(t)) \in L_{r}(C, d Q)$ and

$$
\|\phi(q(0)) \psi(q(t))\|_{r} \leqq\|\phi\|_{2}\|\psi\|_{2}
$$

The $T$ can be chosen independently of $r$ provided $r$ is bounded away from 2.

Proof.

$$
\begin{aligned}
\|\phi(q(0)) \psi(q(t))\|_{r}^{r} & =\int|\phi(q(0))|^{r}|\psi(q(t))|^{r} d Q \\
& =\int|\phi|^{r}\left[\exp \left(-t H_{0 \gamma \gamma}\right)|\psi|^{r}\right] \phi_{\varkappa}^{2} d q \\
& \leqq\left\||\phi|^{r}\right\|_{2 / r}\left\|\exp \left(-t H_{0 \gamma x}\right)|\psi|^{r}\right\|_{p}
\end{aligned}
$$

where $p$ is the conjugate index to $2 / r ;(r / 2)+p^{-1}=1$. However

$$
\||\phi| r\|_{2 / r}=\|\phi\|_{2}^{r}
$$


and

$$
\left\|\exp \left(-t H_{0 \gamma \kappa}\right)|\psi|^{r}\right\|_{p} \leqq\left\||\psi|^{r}\right\|_{2 / r}=\|\psi\|_{2}^{r}
$$

for large $t$, by Lemma 5.1.

\section{§ 7. The Feynman Kac formula}

The Feynman Kac formula states that

$$
\left(\phi, \exp \left(-t H_{\gamma \varkappa}\right) \psi\right)=\int \phi(q(0))^{-} \exp \left(-\int_{0}^{t} V_{\gamma x}(q(s)) d s\right) \psi(q(t)) d Q .
$$

The right member of (7.1) is bounded by

$$
\begin{array}{r}
\|\phi(q(0))-\psi(q(t))\|_{p^{\prime}}\left\|\exp \left(-\int_{0}^{t} V_{\gamma x}(q(s)) d s\right)\right\|_{p} \\
\leqq\|\phi\|_{2}\|\psi\|_{2}\left\|\exp \left(-\int_{0}^{t} V_{\gamma x}(q(s)) d s\right)\right\|_{p}
\end{array}
$$

for $p>2$ and for $t$ large, by Lemma 6.2. Thus

and

$$
\left\|\exp \left(-t H_{\gamma x}\right)\right\| \leqq\left\|\exp \left(-\int_{0}^{t} V_{\gamma \varkappa}(q(s))\right)\right\|_{p}
$$

$$
-t^{-1} \ln \left[\left\|\exp \left(-\int_{0}^{t} V_{\gamma \varkappa}(q(s))\right)\right\| \|_{p} \leqq H_{\gamma \varkappa}\right. \text {. }
$$

Let

Then

$$
I_{\lambda}=\int_{0}^{t} V_{\gamma \lambda}(q(s)) d s
$$

$$
-K_{2} t(\ln \lambda)^{a / 2} \leqq I_{\lambda}
$$

by Lemma 5.2. Let $K_{5}, \ldots$ denote positive constants depending only on $t$ and the polynomial $P$ and let $\operatorname{Pr}$ denote the measure defined by $d Q$. Then

$\operatorname{Pr}\left\{I_{\iota} \leqq-K_{2} t(\ln \lambda)^{d / 2}-1\right\} \leqq \operatorname{Pr}\left\{\left|I_{x}-I_{\lambda}\right| \geqq 1\right\}$

(by Lemma 6.1)

$$
\leqq \int\left|I_{\chi}-I_{\lambda}\right|^{2 j} d Q \leqq t^{2 j}\left\|V_{\gamma \varkappa}-V_{\gamma \lambda}\right\|_{2 j}^{2 j}
$$

(by Lemma 5.3)

$$
\begin{aligned}
& \leqq(d j) ! t^{2 j} K_{3}^{j} \lambda^{-j} \\
& \leqq(d j)^{d j} e^{-d(j+1)} K_{5}^{j} \lambda^{-j}
\end{aligned}
$$

by Stirling's formula. We choose $j$ so that

$$
j \leqq d^{-1} K_{5}^{-1 / d} \lambda^{1 / d}<j+1 \text {. }
$$


Then

$$
e^{-d(j+1)} \leqq \exp \left(-K_{5}^{-1 / d} \lambda^{1 / d}\right)
$$

is a bound for (7.3) and so

Thus

$$
\operatorname{Pr}\left\{I_{\varkappa} \leqq-X-1\right\} \leqq \exp \left(-K_{6} e^{K_{7} X^{2 / d}}\right)
$$

$\int\left|e^{-I_{\boldsymbol{x}}}\right|^{p} d Q=\int e^{-p I x} d Q \leqq e^{-2 p}+\sum_{n \geqq 1} \exp (p(n+2)) \exp \left(-K_{6} e^{K_{7} n^{2 / d}}\right)$

is bounded independently of $\gamma$ and $x$ and combining this with (7.2) we have $H_{\gamma \varkappa}$ bounded below by a constant which is independent of $x$ and $\gamma$; according to $\S 4$, this proves Theorem A.

The formula (7.1) can be proved by standard methods. See for example [6, p. 168-171], where a similar formula is derived.

\section{References}

1. GLimm, J.: Yukawa coupling of quantum fields in two dimensions, I. Commun. Math. Phys. 5, 343-386 (1967).

2. - Yukawa coupling of quantum fields in two dimensions, II. Commun. Math. Phys. 6, 61-76 (1967).

3. Guenin, M.: On the interaction picture. Commun. Math. Phys. 3, 120-132 (1966).

4. Iто, K., and McKeaN, H.: Diffusion processes and their sample paths. New York: Academic Press 1965.

5. JAFFe, A.: Wick polynomials at a fixed time. J. Math. Phys. 7, 1250-1255 (1966).

6. KAC, M.: Probability and related topics in physical sciences. New York: Interscience 1959.

7. LANFORD, O.: Construction of quantum fields interacting by a cutoff Yukawa coupling. Princeton University Thesis 1966.

8. Malgrange, B.: Théorèmes d'interpolation dans les espaces $L^{p}$. In: Seminaire Schwartz, 1959/60. Secretariat mathematique, 11 rue Pierre Curie, Paris.

9. Nelson, E.: Schrödinger particles interacting with a quantized scalar field. In: Analysis in function space, ed. by W. Martin and I. SEgaL, pp. 87-120. Cambridge: M. I. T. Press 1964.

10. - A quartic interaction in two dimensions. In: Mathematical theory of elementary particles, ed. by R. Goodman and I. SegaL, pp. 69-73. Cambridge: M. I. T. Press 1965.

11. - Dynamical theories of Brownian Motion. Princeton: Princeton University Press 1967.

12. Segal, I.: Notes toward the construction of nonlinear relativistic quantum fields, I. The Hamiltonian in two space-time dimensions as the generator of a $C^{*}$-automorphism group. Proc. Natl. Acad. Sci. U. S. 57, 1178-1183 (1967).

13. - Quantized differential forms. (to appear).

Prof. J. GLImM

Department of Mathematics

Mass. Institute of Technology

Cambridge, Mass. USA 